\title{
Anabases
}

ANABASES Traditions et réceptions de l'Antiquité

4 | 2006

Varia

\section{Goffredo Coppola, des études antiques à l'exécution de Dongo}

Philippe Foro

\section{(2) OpenEdition}

1 Journals

Édition électronique

URL : http://journals.openedition.org/anabases/2957

DOI : 10.4000/anabases.2957

ISSN : 2256-9421

Éditeur

E.R.A.S.M.E.

Édition imprimée

Date de publication : 1 octobre 2006

Pagination : 65-75

ISSN : 1774-4296

\section{Référence électronique}

Philippe Foro, «Goffredo Coppola, des études antiques à l'exécution de Dongo », Anabases [En ligne],

4 | 2006, mis en ligne le 01 janvier 2012, consulté le 20 octobre 2019. URL : http://

journals.openedition.org/anabases/2957; DOI : 10.4000/anabases.2957

(C) Anabases 
Anabases 4 (2006), p. 65-75

\section{Goffredo Coppola, des études antiques à l'exécution de Dongo}

PhILIPPE FORO

LE 29 AVRIL 1945, sur la piazza Loreto de Milan, se déroule une scène digne de l'Enfer de Dante. Une foule déchaînée s'apprête à lyncher les cadavres de Benito Mussolini, de sa maitresse Claretta Petacci, de hiérarques exécutés la veille à Giulino di Mezzagra, près de Dongo, avec le Duce. Parmi eux, le corps de Goffredo Coppola, personnage sans doute totalement inconnu de la très grande majorité des personnes présentes ce jourlà. Spécialiste de civilisation gréco-latine, recteur de l'université de Bologne durant la République Sociale, Goffredo Coppola est au nombre de ces intellectuels et universitaires qui participèrent au phénomène, toujours délicat à mesurer mais réel, du consensus (sur environ 1250 universitaires italiens, seuls 12 refusèrent de prêter serment au Duce en $1931^{1}$ ). À partir de 1933, il lui arrive d'écrire dans la revue Vita Nova que dirige Leandro Arpinati, chef du squadrisme à Bologne, podestà (nom donné aux maires à l'époque fasciste) de la ville de 1926 à 1929, avant de devenir sous-secrétaire d'État à l'Intérieur de décembre 1929 à mai 1933. La particularité de Coppola est de s'être engagé le plus ouvertement au moment où le fascisme entre dans sa phase de la plus grande radicalité qui correspond également à son chant du cygne, c'est-à-dire les années 1943-1945. Ce choix politique et sa mort ne doivent pas éclipser le travail intellectuel de Coppola, au travers de son enseignement et de ses publications, ni le fait que Coppola ait servi le régime en souhaitant participer aux guerres engagées par celui-ci, en Éthiopie entre octobre 1935 et mai 1936, dans la Seconde Guerre mondiale à partir de juin 1940. Aussi, la présente contribution propose-t-elle d'analyser le parcours intel-

1 Sur la question de la prestation de serment des universitaires italiens, voir Helmut GOETZ, Il giuramento rifiutato. I docenti universitari e il regime fascista, Milano, La Nuova Italia, 2000. 
lectuel et politique de cet antiquisant à la fois représentatif de la majorité de la communauté intellectuelle italienne au cours de la période fasciste, mais qui s'en écarta lors du choix déterminant de l'engagement au sein de la République Sociale.

\section{Une riche carrière universitaire}

Goffredo Coppola naît le 21 septembre 1898 à Guardia Sanframondi (province de Bénévent, en Campanie) ${ }^{2}$, dans une famille de la moyenne bourgeoisie. De 9 ans à 17 ans, il suit sa scolarité au collège-lycée Giannone de Bénévent. Â la rentrée universitaire de 1915, il s'inscrit au département de langues et littératures grecque et latine de l'université de Naples. En juillet 1920, il soutient sa laurea (examen de licence) sur des fragments de papyrus de poésies de Ménandre. Dans les années 1920, il est assistant de Girolamo Vitelli à l'université de Florence. Ayant obtenu sa libera docenza ${ }^{3}$ en langues et littératures grecque et latine, il est nommé à l'université de Cagliari à la rentrée 1929, puis, deux ans plus tard, il obtient le poste de langue et littérature grecque à l'université de Bologne. Pour la rentrée 1940, il devient titulaire de la chaire de langue et de littérature latine en remplacement du professeur Funaioli (il semble que ce remplacement devait être temporaire car Coppola demande, par une lettre du 4 juillet 1943, à Carlo Alberto Biggini, ministre de l'Éducation nationale, de récupérer la chaire de langue et littérature grecques, comme cela avait été prévu trois ans auparavant).

Grâce à une note manuscrite du 16 janvier 1935, conservée dans le dossier Coppola de l'Archivio centrale dello Stato, on peut suivre les cours dispensés par celui-ci entre 1931 et 1935 et qui font preuve d'un relatif éclectisme.

- 1931-1932 : étude de la pièce d'Aristophane datée de 405 avant J.-C, Les Grenouilles, satire littéraire opposant Eschyle à Euripide ;

2 Les renseignements biographiques et sur la carrière de Coppola sont issus de l'article de Gian Paolo Brizzi, " Goffredo Coppola e l'università di Bologna : uno scomodo caso di continuità istituzionale ", Quaderni di storia, 60 (2004), p. 141-180 et de son dossier conservé à l'Archivio centrale dello Stato à Rome, Min. Pubbli. Istr. Prof. Ordinari (19401970), inv. 16/49 avec un curriculum vitae établi le 16 décembre 1953. Il convient de signaler également la biographie d'Andrea Jelardi, Goffredo Coppola. Un intellettuale del fascismo fucilato a Dongo, Milano, Mursia, 2005, ainsi que la journée d'études consacrée à Coppola à l'université de Bologne, le 12 février 2004. Guido Melis y présenta la République sociale italienne, Gian Paolo Brizzi les archives universitaires bolognaises concernant Coppola, Luciano Casali les aspects politico-scientifiques de ce dernier. Les conclusions furent tirées par Luciano Canfora. Coppola est également un des personnages centraux de son ouvrage intitulé Il papiro di Dongo, publié en 2005 chez Adelphi et qui étudie le destin d'un fragment d'un texte de l'historien Cratippe, continuateur de l'œuvre de Thucydide, La guerre du Péloponnèse, dont le récit s'arrête, on le sait, en 411 avant J.-C. Titre universitaire permettant d'enseigner dans le supérieur. 
- 1932-1933 : étude de l'Agamemnon d'Eschyle qui ouvre, en 458 avant J.-C, la trilogie l'Orestie, l'œuvre présentant le meurtre du roi de Mycènes par Clytemnestre et Égisthe ;

- 1933-1934 : étude de la Politique d'Aristote et d'hymnes et épigrammes de Callimaque (310-235 avant J.-C.) ;

- 1934-1935 : étude des lettres de Saint Paul et des odes de Bacchylide (vers 500 avant J.C), protégé de Hiéron de Syracuse au pouvoir de 478 à 466, et dont les œuvres nous sont connues par des papyrus égyptiens.

Selon un recensement que nous avons effectué à la Bibliothèque nationale de Rome, les travaux de Coppola sont édités entre 1931 et 1943.

- Introduzione a Pindaro, Roma, L'Universale, 1931.

- La poesia d'Alceo, Annali della Facoltà di Filosofia e Lettere di Cagliari, anno academico 1930-1931.

- Polis, manuale di istituzioni pubbliche e private della Grecia, Firenze, Vallechi, 1934.

- L'Orazio di Carducci, Roma, La Nuova Antologia, 1935 ; Documenti del cristianesimo primitivo, Bologna, Zanichelli, 1935 ; Cirene e il nuovo Callimaco, Bologna, Zanichelli, 1935 ; Cimossa Carducciana, Bologna, Zanichelli, 1935.

- Il teatro d'Aristofane, 2 vol., Bologna, Zanichelli, 1936.

- L'erede di Cesare, Bologna, Zanichelli, 1938.

- Il problema della più antica tragedia romana, Firenze, Italia e Grecia, 1939 ; Con la testa sullo zaino, Bologna, Capelli, 1939.

- Il teatro tragico in Roma repubblicana, Bologna, Zanichelli, 1940.

- Augusto. I grandi italiani. Collona di biografie diretta da Luigi Federzoni, Torino, Ed. Torinese, 1941 ; Gaio Lucullo, cavaliere e poeta, Bologna, Zanichelli, 1935 ; Storia della letteratura romana, Bologna, Capelli, 1941.

- Teatro di Terenzio, Bologna, Il Resto di Carlino, 1942.

- La critica neotestamentaria di Erasmo di Rotterdam, Bologna, Zanichelli, 1943.

Dans cette ouvre assez importante, il convient de discerner les publications purement scientifiques et celles qui, tout en gardant une érudition scientifique, participent à l'image que le régime fasciste veut donner de lui vis-à-vis de l'héritage antique. Dans la première catégorie, peut être placé l'ouvrage de 1936 consacré au théâtre d'Aristophane ${ }^{4}$. Travailler sur Aristophane dans le contexte de l'Italie fasciste pose une série de problèmes relatifs aux principes véhiculés dans l'œuvre de l'auteur grec et visà-vis des thèses qui servent de supports idéologiques au régime. Né vers 445 avant J.C. et mort vers 388, Aristophane ne saurait refléter l'esprit militariste et impérial de l'Italie des années 1930. Analysant la relation entretenue par l'auteur grec avec le principe de la paix, Paul Demont écrit : "Platon, et plus encore Aristote, ont fait le même

4 Goffredo Coppola, Il teatro di Aristofane, Bologne, Zanichelli, 1936. 
choix qu'Aristophane et qu'Athènes : c'est la paix, et non la guerre, qui est le but de la vie, c'est donc en vue de la paix, et non en vue de la guerre, qu'il faut administrer un État ${ }^{5}$." Lors de la guerre du Péloponnèse, Aristophane est hostile à une guerre à outrance, demande l'ouverture de négociations entre Sparte et Athènes en 426, est favorable à la paix de 411. Une autre interrogation se pose à propos de l'attitude d'Aristophane vis-à-vis de la cité démocratique de son époque. La tradition en fit un auteur hostile à la démocratie. Les travaux récents prennent souvent le contre-pied de cette interprétation. Ainsi Luciano Canfora dans son Histoire de la littérature grecque : "Rangé parmi les antidémocrates, et même parmi les oligarques pour sa campagne acharnée contre Cléon, Aristophane semblait se contredire par son exaltation du "peuple rameur, rempart de la cité" (Archaniens, 162-163). Les réactions des critiques étaient diverses, depuis ceux qui imaginaient quelque parti “modéré" dont Aristophane eût fait partie (Maurice Croiset, Aristophane et les partis à Athènes), à ceux qui, comme Gomme, désespéraient de connaître les conceptions politiques d'Aristophane, voire concluaient à leur inexistence. [...] En réalité, l'engagement politique d'Aristophane existe [...]. Pour Aristophane, qui, à l'apogée de sa productivité et de sa maturité intellectuelle, a vécu en son entier la longue crise de la démocratie athénienne, le système traditionnel de la cité ne souffre aucune discussion ${ }^{6}$. "

L'ouvrage était prévu en deux volumes. Il semble bien que le second n'ait jamais été publié ce qui est d'autant plus regrettable que, si le premier traite des prédécesseurs d'Aristophane, le second était appelé à traiter du théâtre d'Aristophane lui-même ${ }^{7}$. Le premier volume est divisé en cinq chapitres. Le premier est consacré à Chionidès, Magnétès, Cratetos, le second aux comédies de Cratinos et le troisième analyse la composition des chœurs comiques, en particulier la parodos ${ }^{8}$ et la parabase. Enfin les deux derniers chapitres étudient les dernières productions "cratiniques ", Nemesis et Dyonisos-Alexandre, certainement connues et entendues par Aristophane. Coppola cherche ainsi les origines du théâtre grec, de sa composition, de ses méthodes.

Dans la seconde catégorie est à placer le recueil d'articles, L'erede di Cesare (L'héritier de César), publiés dans Il Popolo d'Italia, journal fondé par Mussolini le 15 novembre 1914 après sa rupture avec le parti socialiste et devenu organe officiel du régime. $Y$ est reprise la tendance fortement romanisante du fascisme. Dans l'article "Pax Augusta " du 17 mars 1937, Coppola évoque la restauration et l'installation

5 Un thème, trois auvres : la paix, Paris, Belin Sup, 2002, p. 84.

6 Luciano CANFORA, Histoire de la littérature grecque d'Homère à Aristote, Paris, Desjonquières, 1994, p. 182.

7 Nos recherches à la bibliothèque nationale de Rome et à la bibliothèque d'histoire moderne et contemporaine n'ont rien donné concernant le second volume consacré au théâtre d'Aristophane. La parodos est le premier chant du chœur après son entrée dans l'orchestra. 
prochaine de l'Ara Pacis d'Auguste dans un bâtiment de la nouvelle piazza Augusto imperatore en voie de construction. Fondé le 4 juillet 13 avant J.-C., l'Autel de la Paix est dédié le 30 janvier 9 avant J.-C. Décoré notamment d'une procession religieuse rassemblant la famille impériale, l'Autel veut célébrer la paix augustéenne et l'épanouissement de l'Italie et du monde sous la domination bienfaisante de Rome. Coppola écrit à ce sujet : "Ceci était la paix d'Auguste, la paix armée de la Rome impériale. C'était le message que Rome envoya aux peuples de la Méditerranée et du Septentrion, annonçant, dans le nom d'Auguste, la grande époque d'une nouvelle civilisation, issue de la victoire et destinée à être une histoire immense et puissante justement parce qu'elle n'était pas seulement une culture mais une civilisation, non seulement une forme mais une action. " Moins d'un an après la proclamation de l'Empire par Mussolini à la suite de la victoire en Éthiopie (9 mai 1936), le rapprochement entre la période d'Auguste et celle du fascisme est aisé. La seconde est héritière de la première. Cette pensée serait à compléter avec l'article du 23 septembre 1937 « La mostra augustea della Romanità " (L'exposition augustéenne de la Romanité). Ce jour-là, le régime fasciste inaugure l'année consacrée au bimillénaire d'Auguste, l'empereur étant né le 23 septembre 63 avant J.-C. "Les historiens médiocres, peu sûrs d'eux, croient que l'Empire romain représente le monde du bien-être matériel et de l'ordre juridique et culturel [...]. Pour nous, fascistes, l'Empire romain est la réalisation de l'unité du monde antique et la synthèse de l'évolution individuelle et collective de l'humanité ; c'est la pacification des conflits, la protection du continent contre la barbarie et la dissolution interne. [...] Quirinio Giglioli et ses collaborateurs [...] ont construit un musée consacré à l'Empire de Rome qui satisfait notre orgueil national, évoque notre très ancienne histoire et exalte la mission universelle de l'Italie. » Le parallèle entre romanité et fascisme va jusqu'à faire remarquer que le castrum légionnaire de Milan se trouve sur la piazza San Sepolcro où furent fondés les Faisceaux de combat en 1919 par Mussolini. L'analogie est pleinement assumée dans un article de Coppola publié le même jour sous le titre "Imperator Caesar Augustus ». " Auguste est le symbole de notre destin et il est comme César une figure éternelle de notre histoire, la plus grande figure contemporaine. Vingt siècles, deux mille ans ne l'ont ni pétrifié, ni diminué : il est présent dans la réalité et dans l'avenir impérial de l'Italie fasciste. " Faut-il être grand clerc pour percevoir qui est le nouvel Auguste?

Dès lors, la biographie d'Auguste est également politique : peut-on écrire dans l'Italie de 1941 une biographie d'Auguste qui ne soit pas politique et sujette à des parallèles avec Mussolini ? Elle est publiée dans une collection dirigée par Luigi Federzoni, un homme qui eut des responsabilités politiques importantes au sein du régime. Membre de l'Association nationaliste en 1910, député en 1913, favorable à la fusion du courant nationaliste dans le fascisme, Federzoni est ministre des Colonies d'octobre 1922 à juin 1924, de l'Intérieur de juin 1924 à novembre 1926 au moment de la mise en place du système dictatorial, à nouveau des Colonies de novembre 1926 à décembre 1928. Sénateur en novembre 1928, il préside le Sénat d'avril 1929 à mars 1939 puis 
l'Académie d'Italie de mars 1938 à la fin du régime fasciste ${ }^{9}$. Ainsi, Coppola publie son Auguste dans une collection éminemment politique. L'empereur y est présenté de manière positive sur le plan personnel. Modeste, affable, d'un « tempérament froid, peu sensible aux attractions sentimentales, Auguste ne fut jamais dominé par les femmes ${ }^{10}$. " En vrai homme d'État, Octave-Auguste ne recule pas devant la nécessité de la force. Ainsi le massacre de Pérouse en 40 avant J.-C permet-il le ralliement à la cause octavienne des Italiens hésitants dans la guerre pour le pouvoir qui suit la mort de César ${ }^{11}$. Si Coppola reconnaît les mérites de ce dernier, il montre en quoi Auguste a su pérenniser l'œuvre césarienne. La créateur de l'Empire fut César mais le fondateur en fut Auguste, lequel a réussi a faire survivre l'œuvre et la gloire de César en cinquantesix ans de règne ${ }^{12}$. [...] Il apparaît garant du miraculeux destin de l'Italie comme dans la formule en usage dans le dernier Empire qui saluait le nouvel empereur avec le souhait qu'il soit plus fortuné qu'Auguste : felicior Augusto 13. Mussolini felicior Augusto?

Si Coppola, parmi d'autres, sert le régime en adhérant à sa vision de l'Histoire, son parcours personnel est également caractéristique d'un membre de la classe moyenne émergente et patriotique.

\section{Un intellectuel patriote et fascisant}

Goffredo Coppola est mobilisé en février 1917 pour servir dans les troupes royales au cours de la Grande Guerre. Après une formation à l'école des officiers de Modène, il sert à partir du 23 septembre 1917 dans la zone d'opérations du Val Posina et sur le Pasubio au sein du régiment d'infanterie Aquila. Son engagement militaire est suffisamment courageux pour qu’il soit décoré de la médaille commémorative de la guerre de 1915-1918, de la médaille de l'Unité de l'Italie, de la médaille interalliée 14. À cet égard, Coppola est assez représentatif des classes moyennes émergentes qui, à la suite du premier conflit mondial, se rallient au fascisme selon l'idée avancée par Renzo De Felice en 1975 : «Le fascisme-mouvement a été l'idéalisation, la velléité d'un certain

$9 \quad$ Signalons que Federzoni fait connaître son opposition aux lois raciales de 1938 et qu'il vote la motion Grandi, lors de la séance du Grand conseil du fascisme du 25 juillet 1943, qui provoque le destitution de Mussolini. Goffredo Coppola, Augusto. I grandi italiani. Collona diretta da Luigi Federzoni, Ed. Torinese, 1941, p. 19.

11 Ibid., p. 55.

12 Remarquons que Coppola fait débuter le règne d'Auguste de 42 avant J.-C., c'est-à-dire de la bataille de Philippes qui vit la défaite des assassins de César.

13 Op. cit., p. 219-221.

14 Ces renseignements sont tirés du document du district militaire de Bologne en date du 7 août 1942 et conservé dans le dossier Coppola du ministère de l'Instruction Publique à l'Archivio centrale dello Stato. 
type de classe moyenne émergente [...] une classe moyenne émergente qui tend à réaliser sa propre politique. [...] Ce fut la Première Guerre mondiale qui mobilisa toute une partie de la société italienne restée jusque-là en déshérence. Et cette partie, mobilisée par la guerre, exclue du pouvoir effectif, de la participation, tend, au travers du fascisme, à revendiquer, à acquérir sa fonction ${ }^{15}$. "Coppola a fort probablement vu dans le fascisme une concrétisation de l'affirmation de l'élévation sociale de la petite et moyenne bourgeoisie dont il est un des membres.

Sa volonté d'engagement patriotique se concrétise, pendant le régime, au moment de la guerre d'Éthiopie. Alors qu'il est professeur de langue et de littérature grecque à l'université de Bologne, il demande à être engagé volontaire, le 25 septembre 1935, à quelques jours du début des opérations. Mais, une lettre du préfet de Bologne signale que le cabinet du Duce " a retourné la demande d'engagement volontaire pour l'Afrique Orientale 16 ", ce que confirme une lettre du ministère de l'Éducation nationale du 29 novembre 1935. Goffredo Coppola ne participe donc pas à la conquête de l'Empire. Par contre, il est rappelé sous les drapeaux, le $1^{\text {er }}$ avril 1939, comme capitaine au

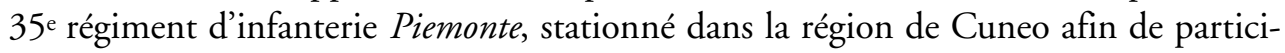
per aux préparatifs en vue d'un éventuel conflit avec la France. L'inaction qui suit la proclamation de la non-belligérance de l'Italie au début de la Seconde Guerre mondiale, lasse le capitaine Coppola qui obtient de réintégrer son poste à l'université de Bologne en novembre 1939. Un nouveau départ sous les drapeaux suit la déclaration de guerre du 10 juin 1940 à la France et à la Grande-Bretagne. Après avoir séjourné dans les Alpes jusqu'au 25 août ${ }^{17}$, l'unité du capitaine Coppola est destinée à partir en Libye d'où les troupes du marchal Graziani préparent une offensive contre les forces britanniques stationnées en Égypte. Coppola fait une intervention auprès du recteur de son université, Alessandro Ghigi, afin de revenir sur son lieu d'enseignement, ce qui lui est accordé. La carrière militaire de Goffredo Coppola aurait pu s'arrêter là. Or, le 16 mai 1942, il demande son affectation dans la $8^{\mathrm{e}}$ armée italienne qui se bat en Union soviétique ${ }^{18}$. Arrivé sur le front de l'Est le $1^{\text {er }}$ juillet, sa bonne connaissance de la langue allemande permet de lui confier une mission de liaison auprès des troupes du Reich. Blessé suite à des gelures, Coppola est rapatrié de Russie, le 19 novembre, au moment où les forces soviétiques lancent l'offensive qui aboutit à l'encerclement de la

15 Renzo De Felice, Intervista sul fascismo, Roma-Bari, Laterza, 1997 (3e édition), p. 30-31.

16 Document conservé dans le dossier Coppola déjà signalé.

17 C'est un document de l'université de Bologne en date du 17 février 1942 qui précise que Goffredo Coppola a effectué une période militaire sur le front des Alpes du 13 juin au 25 août 1940. Elle est conservée dans le dossier Coppola du ministère de l'Instruction Publique à l'Archivio centrale dello Stato.

18 Ceci nous est connu par un courrier du directeur général des affaires universitaires de l'université de Bologne, Giuseppe Giustini, en date du 23 mai 1942. L'Italie fasciste déclare la guerre à l'Union soviétique à la suite de l'agression allemande (opération Barbarossa) du 22 juin 1941. 
$\sigma^{e}$ armée allemande à Stalingrad et à l'écrasement de la 8 e armée italienne dans la boucle $\mathrm{du}$ Don. Cette blessure amène les autorités militaires à le déclarer invalide de guerre, le 16 juin 1943. Auparavant, il avait repris en janvier son enseignement de langue et littérature latines.

Cet engagement de Coppola ne manque pas d'interroger. À 44 ans, aller combattre sur le terrible front de l'Est n'est pas le choix de la facilité. Alors pourquoi ? Une lettre de Coppola au recteur Ghigi permet d'avancer une hypothèse. Cette correspondance est du 25 octobre 1942. "Ceci nous conforta tous dans l'idée que ces bêtes bolcheviques finiront comme doivent finir les sans Dieu. Non pas les gens qui ont toujours souffert mais leurs chefs qui les ont dominés par une méthode brutale que vous ne pouvez imaginer, cher Recteur. [...] J'ai vu les églises, toutes les églises d'ici, réduites, devenus des dépôts, toutes, sans aucune exception. Quand nous avons distribué figurines de saints et images du Christ, vieux et jeunes accouraient comme des assoiffés et des affamés. Ceci est une des choses que j'ai vues parmi tant d'autres et toutes ces choses m'ont profondément impressionné ${ }^{19}$." L'anticommunisme de Goffredo Coppola est alors peut-être un des moteurs de son engagement politique plus radical.

\section{L’engagement dans la République Sociale}

Arrêté à la suite de sa destitution du 25 juillet 1943, Mussolini est libéré par un commando de parachutistes allemands, le 12 septembre, de sa prison du Gran Sasso d'Italia, point culminant des Apennins. Après un entretien avec Hitler à Munich, il accepte de fonder, le 18 septembre, un nouveau régime fasciste dans le nord de l'Italie, la République Sociale italienne, dont les autorités gouvernementales s'installent à Salò, sur les bords du lac de Garde. Avec une volonté de retrouver une "pureté » originelle, la République Sociale entame la période la plus radicale du fascisme ${ }^{20}$. Or, c'est à ce moment-là que Goffredo Coppola choisit de servir la République Sociale. Après être devenu pro-recteur le 24 novembre 1943, il est élu recteur de l'université de Bologne, le 18 décembre, et le reste jusqu'à l'effondrement de celle-ci et de la domination allemande en Italie du nord à la fin avril 1945.

Pourtant jusqu'au milieu des années 1930, l'engagement de Goffredo Coppola au sein du régime fasciste est réel mais il ne figure pas parmi les ultras. Avec le décès de son maitre Girolamo Vitelli en 1935, qui a pu avoir une influence modératrice, cet engagement se fait plus actif. Il est vrai que le département des Lettres compte parmi

19 Cité in Gian Paolo BrizZI, op. cit., p. 151-152.

20 Parmi l'historiographie de la République Sociale italienne, on peut retenir Aurelio Lepre, La storia della Repubblica di Mussolini. Salò : il tempo dell'odio e della violenza, Milano, Mondadori, 1999 ; Luigi Ganapini, La repubblica delle camicie nere, Milano, Garzanti, 2002. 
les plus alignés sur les volontés du régime. Les travaux de Gian Paolo Brizzi ont montré que le tiers des professeurs épurés à la fin de la guerre au sein de l'université de Bologne est issu du département des Lettres et que les lois raciales de 1938 aboutissent à l'exclusion de 51 professeurs, docteurs et assistants ${ }^{21}$. Comme nous avons pu le constater avec ses analyses de la romanité, la fin des années 1930 et la guerre forment la période du plein engagement de Coppola. Arrêté un temps par le gouvernement Badoglio 22 pour apologie du fascisme, Coppola, une fois libéré par les Allemands le 9 septembre, s'engage dans la République Sociale avec la ferme volonté de ne pas dévier, ni trahir le Duce. Il s'en explique dans le bimensuel L'Assalto du 15 octobre 1943 : « Nous déclarons à visage découvert notre foi républicaine de fascistes dévoués au Duce, de combattants qui ont toujours servi avec honneur, d'hommes de culture qui n'ont jamais fait de la chaire une tribune de laquelle seraient prononcées des paroles qui ne seraient pas en conscience, et jusqu'au sacrifice, une loi morale. " Il semble bien qu'il soit nécessaire de prendre en compte dans l'engagement actif de Coppola dans le fascisme de la République Sociale, la volonté de réagir vis-à-vis de ce qui est considéré comme trahison et bassesse. Il y a comme la volonté de sauver un honneur qu'auraient mis à mal la " trahison " du 25 juillet et l'armistice du 3 septembre 1943 entre le gouvernement Badoglio et les Alliés. Le 23 novembre, Carlo Alberto Biggini, redevenu ministre de l'Éducation nationale ${ }^{23}$, le nomme pro-recteur, puis recteur, à la suite d'un vote du Conseil d'administration de l'université. Une des premières décisions du recteur est de suspendre les bourses d'études pour les étudiants, ce qui favorise objectivement l'incorporation de jeunes dans les forces armées de la République Sociale, constituées par la loi du 27 octobre 1943 et basées sur la conscription. D'autre part, Coppola apparaît comme un interlocuteur viable vis-à-vis des autorités d'occupation, tant sur le plan politique qu'en raison de sa très bonne connaissance de la langue allemande. Il porte une lourde responsabilité morale dans les événements du 20 octobre 1944 qui voient les Allemands, appuyés par des auxiliaires fascistes, prendre d'assaut la bibliothèque de Géographie de l'université où se trouvaient de jeunes résistants, les cadavres des morts étant exposés une journée au sein de l'établissement universitaire. À aucun moment, Coppola ne proteste. Pire! Le 23 octobre, à Vérone, le SS-Sturmbannführer, Ernst

21 Gian Paolo BRIZZI, Silence and Remembering. The racial Laws and the foreign Jewish Students at the University of Bologna, Bologna, Il Mulino, 2002.

Suite à la destitution de Mussolini, le roi Victor-Emmanuel III confie au maréchal Pietro Badoglio le soin de former un nouveau gouvernement qui est secrètement chargé de négocier un armistice avec les Alliés. Il est signé à Cassibile, en Sicile, le 3 septembre 1943. Juriste, professeur à Sassari de 1932 à 1938, puis à Pise, Carlo Alberto Biggini avait été recteur de cette université en 1941 et jusqu'à sa nomination au ministère de l'Éducation nationale en février 1943. Ayant voté contre la motion Grandi lors de la séance du Grand Conseil du fascisme du 25 juillet 1943, il avait retrouvé son poste ministériel lors de la constitution de la République Sociale. Très malade, il meurt à l'hôpital de Padoue en 1945. 
Turowski, ordonne que les officiers allemands soient requis, si nécessaire, pour la protection du professeur Coppola. Deux jours plus tard, il est admis comme collaborateur de la SS. Désormais, l'engagement de Coppola est total et ne peut que difficilement laisser de porte de sortie 24 .

Au cours de l'existence de la République Sociale, les contacts du recteur de l'université de Bologne avec le Duce sont réguliers. Selon un décompte des demandes d'audiences accordées, Coppola rencontre quinze fois Mussolini entre le 4 mars 1944 et le 5 avril $1945^{25}$. L'objectif du recteur est de présenter les publications de la revue Civiltà fascista dont le premier numéro est annoncé à Mussolini dans une lettre du 3 avril 1944 où il évoque également le programme de l'Institut national de culture fasciste à la tête duquel il succède en remplacement de Giovanni Gentile, abattu par des partisans le 15 avril, près de Florence. Les thèmes de la revue correspondent à la ligne défendue par le régime. Ainsi, dans un courrier du 11 juin 1944, Coppola évoque pour le prochain numéro des articles sur les raisons de l'entrée en guerre de l'Italie en 1940, la socialisation, le parti unique, l'État intégral. Dans le numéro d'août, on trouve des articles culturels tel celui consacré à l'historien romain Tite-Live ou celui qui étudie Mazzini et la poésie. Le mois suivant, aux côtés d'une étude sur la philosophie allemande, Coppola revient sur les raisons et les événements de cinq années de guerre. À la suite d'un séjour en Allemagne, il écrit au Duce le 20 février 1945 : "J'ai des documents assez sérieux et importants que je crois de mon devoir de soumettre à votre attention. " En l'occurrence, il s'agit sans doute de nouvelles à propos des armes secrètes allemandes, ultime et chimérique espoir de victoire pour les nazis et les fascistes de la République Sociale.

La fin de Goffredo Coppola est liée à celle de Mussolini. Le 21 avril 1945, il est démis de ses fonctions de recteur au profit du professeur Felice Battaglia désigné par le Comité de Libération Nationale. Il quitte alors Bologne pour Milan. Le 25 avril, Mussolini et ses derniers fidèles quittent la capitale lombarde après d'infructueuses négociations menées sous l'autorité du cardinal-archevêque, Mgr Schuster, avec des représentants du Comité de Libération nationale de la Haute Italie. Après un passage à Côme, Coppola rejoint Mussolini à Menaggio, sur la route de la Suisse. Arrêtés le 27 avril par des partisans, les membres du groupe qui accompagnent celui qui fut le Duce de l'Italie fasciste sont exécutés, le 28, à Giulino di Mezzagra, à côté de la commune de Dongo ${ }^{26}$. À la lecture de l'article 5 du décret du 25 avril du Comité de Libération nationale, Coppola aurait pu ne pas être exécuté : « Les membres du gouvernement fasciste et les hiérarques du fascisme, coupables d'avoir contribué à la suppression des garanties constitutionnelles, détruit les libertés populaires, créé le régime fasciste, compromis et trahi le pays et de l'avoir conduit à l'actuelle catastrophe, sont 
punis de la peine de mort. » Or, il est délicat de faire tomber l'ex-recteur de l'université de Bologne sous ces chefs d'accusation. Mais, dans le contexte et l'atmosphère de guerre civile, finement étudiés par Claudio Pavone 27, le colonel Valerio (Walter Audisio) ne fait pas de détails. Coppola paye donc de sa vie le choix de suivre Mussolini. Les corps sont exposés sur la piazza Loretto de Milan le lendemain. La dépouille de Goffredo Coppola est placée dans le carré $\mathrm{n}^{\circ} 16$ du cimetière Musocco, entre Achille Starace, secrétaire du Parti National fasciste de décembre 1931 à octobre 1939 et Fernando Mezzasoma, ministre de la Culture de la République Sociale. Une lettre du 28 juillet 1951 du recteur de l'université de Bologne informe Antonio Segni, ministre democrate-chrétien de l'Instruction Publique, que les restes mortels de Coppola ont été transférés dans la tombe familiale 28 .

PHILIPPE FORO

Université de Toulouse-Le Mirail

UFR d'Histoire, Arts et Archéologie

5, allées Antonio Machado

31058 Toulouse cedex 9

philippe.foro@wanadoo.fr

27 Claudo Pavone, Una guerra civile. Saggio storico sulla moralità nella Resistenza, Torino, Bollati Boringhieri, 1991. Traduction française, Une guerre civile. Essai historique sur l'éthique de la Résistance italienne, Paris, Seuil, 2005.

Archivio centrale dello Stato, Ministero della Pubblica Istruzione, Professori ordinari (1940-1970), inv. 16/49. 